\title{
Protée
}

\section{Support et iconicité, ou l'apparence sans qualités}

\section{Anne-Marie Christin}

Volume 26, numéro 3, 1998

Logique de l'icône

URI : https://id.erudit.org/iderudit/030528ar

DOI : https://doi.org/10.7202/030528ar

Aller au sommaire du numéro

Éditeur(s)

Département des arts et lettres - Université du Québec à Chicoutimi

ISSN

0300-3523 (imprimé)

1708-2307 (numérique)

Découvrir la revue

Citer cet article

Christin, A.-M. (1998). Support et iconicité, ou l'apparence sans qualités. Protée, 26(3), 69-76. https://doi.org/10.7202/030528ar

\section{Résumé de l'article}

À partir de remarques de Peirce prouvant qu'il était sensible à la "qualité » de certains supports, on s'interroge sur le fait qu'il n'ait pas pris en compte cette qualité dans sa conception de l'« icône ". L'hypothèse avancée ici est que cette ignorance - ou ce refus - a son origine dans la définition que Platon avait donnée luimême de l'icône, et qui est fondée sur l'amalgame d'interprétations erronées relatives à la peinture et à l'écriture. La notion de " représentation », notamment, est à l'origine de confusions qui ont interdit à Platon - puis à Peirce - de voir que la différence essentielle qui sépare idéogramme et lettre alphabétique repose sur la relation fonctionnelle que l'un entretient, et l'autre non, avec son support. 


\title{
SUPPORT ET ICONICITÉ, OU L'APPARENCE SANS QUALITÉS
}

ANE-MREF C CHESTIN

\begin{abstract}
Veuillez excuser le papier sur lequel j'écris. Je viens de m'apercevoir, à mon grand étonnement, que je n'ai plus que ce papier qui ne convient vraiment pas pour vous écrire; et il est trois heures et demie du matin et je suis à cinq ou six miles du papetier le plus proche.
\end{abstract}

Ainsi commence la lettre que Peirce écrit à Victoria Lady Welby le 14 décembre $1908^{1}$. Le regret qu'exprime le philosophe de ne pouvoir disposer du papier qui aurait convenu à sa lettre n'est assurément pas de simple politesse. On ne peut douter qu'il existe à ses yeux certains papiers qui, mieux que d'autres, se prêtent à la correspondance. Et le fait est que peu d'objets possèdent une aussi grande vertu «dynamoïde», pour utiliser le vocabulaire de Peirce, que le papier dit «à lettres»: censé inciter le scripteur à formuler sa pensée de telle manière qu'elle puisse s'ajuster à l'avance à celle de son destinataire, il doit constituer aussi le relais intime et sensible qui les unira l'un à l'autre au-delà du message qu'il contient.

Doué d'un pouvoir de suggestion aussi subtil, le papier à lettres est un objet ambigu, dont les effets peuvent surprendre ou charmer. Peirce nous le prouve d'ailleurs dans cette même lettre, lorsqu'il confie à Lady Welby dans les lignes qui suivent celles que je viens de citer: "Je ne peux vous dire combien j'ai été heureux samedi dernier de voir votre écriture sur l'enveloppe». L'enveloppe appartient à la même catégorie d'objets que le papier à lettres - les fournitures d'écritoires les proposent du reste toujours ensemble et assortis - mais elle en diffère cependant, car elle est destinée à recevoir un message unique et stéréotypé: le libellé d'une adresse. Elle en diffère également parce que sa fonction est de dissimuler dans ses plis un second texte, qui justifie son envoi, celui de la lettre elle-même. On observera enfin que l'adresse d'une enveloppe ne se trouve jamais rédigée à l'intention de son destinataire effectif mais d'un intermédiaire anonyme, sorte de destinataire provisoire dont on sait qu'il prêtera seulement attention à l'information qu'elle délivre en ignorant ses aspects formels et leur message indéfinissable et muet. L'enveloppe d'une lettre constitue donc, en dépit de sa structure contrainte et anodine, un objet plus insolite encore que son papier, puisqu'elle implique l'intervention successive de trois partenaires différents l'auteur, le porteur de la lettre, et son destinataire - et qu'elle combine deux 
éléments hétérogènes l'un à l'autre, mais qui ne peuvent remplir chacun leur fonction qu'à travers leur relation mutuelle et la référence qu'ils font ensemble au document qu'elle contient: un support l'enveloppe proprement dite - et une image - l'adresse qui s'y trouve inscrite (ou dont elle attend l'inscription).

Lorsque Peirce avoue à Lady Welby le bonheur qui a été le sien en reconnaissant son écriture sur l'enveloppe d'une lettre, il fait état d'une expérience familière à beaucoup d'entre nous, mais à laquelle nous ne sommes pas tous aussi vivement sensibles que lui. Il est d'autant plus surprenant de constater qu'il évoque cette expérience de façon superficielle, et en partie inexacte. Sans doute est-il conscient du fait que ce n'est pas d'identifier simplement sur l'enveloppe son nom et son adresse qui a suscité son émotion mais l'écriture dans laquelle il les lisait - autrement dit leur image. Cependant la définition qu'il donne de cette image en occulte la complexité. Selon lui, en effet, une écriture peut être isolée de l'enveloppe sur laquelle elle apparaît, comme une «figure» l'est de son «fond». Or il semble difficile de réduire l'image d'une écriture à sa seule figure graphique. En premier lieu parce que cette figure est elle-même nécessairement composite. Non seulement, en effet, chacun de ses mots résulte d'un assemblage de lettres qui constituent individuellement autant de "figures" distinctes, mais c'est la structure complète de l'adresse, l'ensemble de mots qui la composent, qui lui donne sa valeur d'image. Comment alors déterminer les critères nécessaires à la définition de cette unité? Et de quelle nature sont les indices qui ont permis à Peirce de reconnaître dans cette écriture parmi d'autres l'écriture-de-Lady-Welby, c'est-à-dire un style graphique suffisamment autonome par rapport aux textes où il s'incarne pour qu'il puisse lui apparaître comme la marque identitaire de Lady Welby?

À ces difficultés s'en ajoute une autre qui, à mon sens, est la principale. Car il est impossible de définir le graphisme de Lady Welby, en ce qu'il a de spécifique, sans prendre également en compte le style de sa mise en page. Or c'est ce qu'interdit a priori la formule qu'utilise Peirce, lorsqu'il dit qu'il a reconnu cette écriture «sur l'enveloppe» d'une lettre, comme si elles se trouvaient indépendantes l'une de l'autre. Cette formule peut évidemment se justifier par le caractère marginal d'une remarque aussi intime située dans le contexte d'un courrier dont le propos est philosophique avant tout. Il me semble néanmoins qu'elle constitue déjà un symptôme significatif de la manière dont Peirce tient à laisser à l'écart de sa définition de l'icône ce qui a trait à son support. Si, en effet, l'écriture de Lady Welby peut être considérée comme un indice de sa personne, c'est à la fois parce que le tracé de ses lettres comporte certains effets de plume qui leur sont particuliers, mais aussi parce qu'il existe entre les mots et les lignes ainsi tracés un rythme d'espacement qui en est indissociable. Or un tel rythme ne vient pas à une écriture par son graphisme mais par ce qui l'environne et la détermine comme texte: il naît du blanc du papier.

Du blanc, et non du papier. Ce qui, en effet, importe surtout, dans le papier d'une lettre comme dans celui de tout autre écrit intentionnel - poème ou calligraphie -, n'est pas sa matière proprement dite, mais le fait qu'elle ait été choisie par le scripteur, c'est-àdire que celui-ci lui ait reconnu les qualités indispensables à son projet d'écriture. Ces qualités sont de deux ordres. La première a trait effectivement à la texture du papier, mais dans la mesure où cette texture doit satisfaire à une exigence précise, qui est d'offrir au scripteur un support assurant à sa main, lorsqu'elle écrit, la prise dont elle a besoin, prise où entrent en compte à la fois ses habitudes gestuelles et la nature de l'instrument dont elle se sert. Paradoxalement, si sa matière joue un rôle dans le choix d'un papier à écrire c'est par la capacité qu'elle peut avoir de se faire oublier, de disparaitre, ou de participer de façon «amoureuse» à l'élaboration du texte (c'est ainsi que l'on dit de certains papiers d'imprimerie qu'ils sont "amoureux de l'encre»). L'autre qualité requise d'un papier concerne son apparence visuelle: un blanc plus ou moins rompu, une intensité de couleur plus ou moins forte, etc. La nuance élue par le scripteur doit contribuer en effet à 
faire de ce papier, lorsqu'il l'aura sous les yeux, le lieu d'attente et d'accueil du texte à venir, l'invite à construire dans son espace l'architecture de mots d'où il verra naître sa pensée. Mais aussi - puisqu'il s'agit ici d'un papier choisi pour la correspondance - cette couleur aura valeur d'écran, de miroir au-delà duquel sera présente la figure imaginaire de l'autre, du destinataire de la lettre, dont on sait qu'il substituera son regard à celui de l'épistolier sur cette même page en voie d'écriture.

C'est sa qualité d'apparence antérieure à toute trace qui fait l'iconicité du support d'un texte ou d'une image. Cette iconicité n'opère pas à la façon de l'objet impulsant de l'extérieur sa ressemblance à une figure, mais de l'intérieur même du support, sa surface ayant seule pouvoir de susciter, par le contexte d'imminence où elle l'inscrit, la venue au visible de cette figure. Peirce ne saurait être tenu pour responsable de la mise à l'écart de cette qualité dans sa conception de l'iconicité: comme nous venons de le constater, il y était le premier sensible. Cette exclusion remonte en fait à la définition de l'«icône», telle que nous la devons à Platon. C'est donc à elle qu'il nous faut revenir, non seulement parce que l'ignorance du rôle du support dans l'image y est en effet centrale, mais parce que c'est l'analyse des motifs d'une telle ignorance qui pourra nous éclairer sur la contradiction singulière qui gouverne le premier niveau de la sémiotique de Peirce.

L'icône platonicienne ne saurait avoir de support pour trois raisons. La plus fondamentale est que, selon Platon, l'icône ne représente pas un objet réel mais une idée, et qu'elle-même ne relève pas du visible matériel, comme l'«idole», mais de l'imaginaire et de la pensée. Cette transcendance iconique n'a pas été conçue par lui sur la base d'une quelconque observation, elle est le produit d'un raisonnement posant en prémisse l'existence d'un exercice idéal de la vue, lequel aurait servi ensuite de modèle à la vision matérielle. C'est ainsi que, dans le Timée, la création du monde nous est décrite comme résultant d'une vue divine, dont la nôtre serait la conséquence dégradée $^{2}$. Ce raisonnement prend appui sur une tradition métaphysique très ancienne et dont procède la divination - à laquelle d'ailleurs Platon fait également référence dans ce même texte ${ }^{3}$. Le deuxième motif, au nom duquel Platon refuse tout crédit au support iconique, a sa source dans une théorie de la vision, elle aussi générale au monde antique, selon laquelle notre regard émettrait selon les uns un "rayon visuel», un «feu parfaitement pur" ${ }^{4}$, pour atteindre les objets qu'il veut voir. Cette théorie a pour conséquence de nous faire considérer toute surface qui fait obstacle à ce rayon, ou le brise, comme dénaturant non seulement la vision elle-même mais la vérité de son objet ${ }^{5}$. Pouvant se trouver ainsi à l'origine d'illusions gravement trompeuses, la surface des apparences ne saurait être que suspecte, sinon condamnable par principe. Enfin, et d'un tout autre point de vue, la conception platonicienne de l'icône se fonde sur la vertu des noms, qui leur permet de libérer du réel le monde des choses visibles et de combiner leurs figures dans des créations imaginaires et symboliques: la métaphore et la comparaison - telle celle que fait Alcibiade de Socrate avec les statues de Silène dans Le Banquet ${ }^{6}$ - constituent des icônes au même titre que les visions idéales: il semblerait même qu'elles aient droit de façon plus légitime encore à une telle appellation, le visible en son essence ne pouvant nous devenir accessible par le visible luimême, mais à travers les mots qui le suggèrent.

Ce déplacement du raisonnement de l'ordre du visible vers un autre, qui lui est étranger, afin de rendre mieux compte de ce qui le définit comme tel, ne manque pas cependant de surprendre. Pourquoi avoir fait du langage le véhicule privilégié de l'icône, alors qu'il semble ne pouvoir nous communiquer que l'analogie d'une figure ou d'une chose, c'est-à-dire une information indirecte à leur sujet, et que le visible, quel qu'il soit, relève au contraire de l'évidence? Sans doute cette médiation devait-elle paraître naturelle et nécessaire à Platon. Et le fait est que la conception esthétique qui s'était imposée à son époque reposait exclusivement sur la notion de "représentation", autrement dit sur une interprétation elle-même analogique du réel. Il est certain que, vue sous cet 
angle, la référence indirecte qui caractérise la langue peut dégager la figure de l'illusion où aurait pu risquer de l'entraîner - et l'entraîne selon Platon - la représentation picturale, laquelle combine à une conception dégradée de cette figure - puisqu'elle la matérialise - l'ambiguité foncière de son support. "J'appelle images, dit Platon dans le livre VI de La République, d'abord les ombres, ensuite les reflets que l'on voit dans les eaux, ou à la surface des corps opaques, polis et brillants, et toutes les représentations semblables" ?. Si «l'idole» - qui est l'image à laquelle il est évidemment fait allusion ici - doit être opposée à «l'icône», c'est d'abord parce qu'elle dépend de façon étroite de son support, plus encore que par son caractère matériel. La description, que l'on trouve dans ce même livre VI, de la manière dont les philosophes peuvent construire le plan de la cité idéale est très instructive à cet égard ${ }^{8}$. Elle nous confirme que Platon connaissait bien la peinture et savait que son premier principe réside dans la possibilité qu'elle offre de transposer sur une surface plane la figure des objets, et sa principale difficulté dans le moyen d'y parvenir. Mais il ne se soucie d'y faire référence que pour montrer combien peut s'avérer tâtonnante l'élaboration de figures dont on veut qu'elles soient idéales, sans aborder la question du support. Or si la nature de son support est à l'origine des difficultés de l'art de peindre, elle l'est aussi, et d'abord, du résultat auquel elle lui permet de parvenir, et qui est de faire de ses figures autant de révélations, c'est-à-dire d'images capables de proposer, fussent-elles tout à fait réalistes, un événement visuel aussi puissant que celui même de l'idéal.

Mais c'est un faux procès que Platon veut faire à la peinture. Il semble lui reprocher d'avoir tué en elle son pouvoir de révélation en se donnant pour seul but celui de la représentation. Mais c'est aussi que le philosophe ne peut concevoir de messages autrement que créés et transmis par la parole et seulement par elle. Telle est la leçon profonde, à mon sens, de l'allégorie de la caverne qui ouvre le livre VII de La République. Cette mise en scène étrange d'hommes enchainés depuis l'enfance dans une grotte et condamnés à regarder devant eux sans pouvoir s'observer les uns les autres tandis que défile, sur la paroi de la caverne leur faisant face, l'ombre projetée par un feu, situé à l'extérieur, d'«hommes portant des objets de toute sorte [...] et des statuettes d'hommes et d'animaux, en pierre, en bois, et en toute espèce de matière", nous signifie que l'image, envisagée cette fois dans sa dualité de "fond" et de "figures", d'espace et de représentation, ne saurait être un instrument de connaissance. Mais Platon veut nous faire comprendre aussi qu'elle n'est pas non plus un moyen de communication. Une telle fonction est réservée de façon exclusive à la parole. Il le suggère de deux manières. En faisant de la paroi de la caverne un lieu de dépôt, non de création, des formes visuelles, et en complétant son récit par des indications que hante de façon constante la communication verbale. Décrivant les personnages qui défilent, il précise en effet: «Naturellement, parmi ces porteurs, les uns parlent et les autres se taisent", et il reprend un peu plus loin, évoquant cette fois les spectateurs: «[...] si la paroi du fond de la prison avait un écho, chaque fois que l'un des porteurs parlerait, croiraient-ils entendre autre chose que l'ombre qui passerait devant eux?»9.

Ce que reproche Platon à la peinture n'est pas de pouvoir conduire à la magie - acception qui est pourtant celle que nous avons retenue d'abord du mot «idole»-, c'est d'être incapable de transmettre, et plus encore de produire, un message verbal. Aussi la question que nous devons nous poser est-elle plutôt de savoir si ce n'est pas en réalité, à travers la peinture, l'écriture que condamne Platon. En quoi, en effet, la comparaison qu'Alcibiade fait de Socrate avec une statue de Silène peut-elle être spécifique d'une «icône», sinon parce que Platon la considère par principe comme relevant de l'oralité, c'est-à-dire comme un produit direct de l'imaginaire et de la parole, à la différence de l'image, assurément, mais d'abord et surtout du texte écrit? Or il s'agit là d'une définition qui ne manque pas d'être arbitraire, puisque nous savons que certains textes de description visuelle, en particulier l'«ekphrasis», bien que lointainement inspirés du modèle homérique, ne se 
sont constitués comme des genres littéraires que par l'écrit. La fragilité de l'argument nous en révèle toutefois d'autant plus l'importance aux yeux de Platon. C'est moins, en effet, la structure du texte qui le préoccupe que le fait qu'il puisse naître écrit, l'écriture représentant pour lui un medium qui dénature la parole, comme le fait un miroir du rayon visuel, en obligeant cette parole à s'inscrire sur une surface trompeuse, aide-mémoire supposé mais qui trahit la mémoire, et la pensée elle-même, et qui les asservit l'une et l'autre aux lois suspectes et vaines de la lecture.

Le Phèdre nous confirme cette assimilation de l'écriture à la peinture en nous livrant aussi de nouveaux motifs de la méfiance de Platon envers l'écrit. Dans ce dialogue, Platon prend cette fois en considération les deux composantes - figures et support - de la peinture, et en insistant de façon toute particulière, à l'opposé de ce que l'on constate dans l'allégorie de la caverne où la paroi de la grotte ne joue qu'un rôle accessoire, sur le second. Il ne le présente plus seulement, en effet, comme un facteur d'illusions, mais comme un traître au discours et à ses fonctions fondamentales. Pour reprendre une formule de Jean-François Lyotard qui témoigne de la permanence du point de vue de Platon jusqu'à nos jours: «La parole implique la co-présence du locuteur et du récepteur. Supprimez la présence du locuteur, vous avez l'écriture; le trait pertinent est le rapport du sujet et du discours» ${ }^{10}$. Voilà ce qui scandalise Platon dans l'écriture. Absence de locuteur, absence d'auditeur aussi: elle ne peut conduire au savoir, non seulement parce que les traces de ce savoir y sont douteuses, mais parce que son support, hérité de la peinture, l'a condamnée au silence:

C'est que l'écriture, Phèdre, a un grave inconvénient, tout comme la peinture. Les produits de la peinture sont comme s'ils étaient vivants; mais pose-leur une question, ils gardent gravement le silence. Il en est de même des discours écrits. On pourrait croire qu'ils parlent en personnes intelligentes, mais demande-leur de t'expliquer ce qu'ils disent, ils ne répondront qu'une chose, toujours la même. Une fois écrit, le discours roule partout et passe indifféremment dans les mains des connaisseurs et dans celles des profanes, et il ne sait pas distinguer à qui il faut, à qui il ne faut pas parler. 11

On ne peut comprendre ni discuter une telle condamnation de l'écriture sans la situer dans le contexte de l'époque où elle est formulée. Au 4e siècle avant notre ère, en Grèce, l'écriture alphabétique constitue encore un mode de communication et d'expression dont l'usage littéraire est récent, et qui n'est nullement parvenu à affaiblir une tradition de pensée reposant sur l'oralité. Elle le pouvait d'autant moins qu'elle-même avait introduit dans l'histoire de l'écriture une rupture radicale au bénéfice de l'oral, chacune de ses lettres renvoyant à un son distinctif de la langue, voyelle ou consonne, ce qu'aucun des systèmes qui le précédaient - idéographique ou sémitique - n'avait permis jusqu'alors. L'écriture idéographique était d'autre part toujours visible sur les monuments égyptiens, et ceux des Grecs qui avaient séjourné en Égypte - tel Platon lui-même avaient pu observer le caractère apparemment pictural des hiéroglyphes, qui semblait autoriser à en interpréter les signes comme autant de représentations figuratives. Les Grecs ignoraient tout, cependant, des principes de fonctionnement de l'écriture qui leur correspondait: aussi leur était-il impossible de concevoir à quel point un tel système différait du leur.

La conséquence de cette situation est que l'on pouvait sans doute considérer légitimement, du temps de Platon, qu'il n'existait qu'un seul type d'écriture - l'alphabet -, et que celui-ci était une représentation de la parole, mais dans la mesure où l'on opérait ainsi un amalgame entre des données profondément différentes les unes des autres, et qui les faussait chacune. Il n'était pas possible, en effet, d'affirmer que l'alphabet était une représentation de la parole sans constater simultanément que ce qu'il permettait de représenter était en fait le squelette abstrait de cette parole, ses "éléments", comme le dit Platon dans le Théétète ${ }^{12}$. Et lui-même d'ailleurs observe tout le premier que ces éléments ne conduisent à aucune prononciation verbale effective, 
à la différence des syllabes qu'ils permettent pourtant de composer ${ }^{13}$. L'écriture alphabétique est, à la vérité, un système foncièrement incohérent, où la fonction de représentation que l'on peut lui reconnaître n'a pas de valeur référentielle, contrairement aux figures d'un tableau, mais constitue un simple code. Cette notion de représentation appliquée à l'écriture devait certainement beaucoup à l'apparence réaliste des hiéroglyphes. Mais elle résultait surtout d'un contresens relatif à la nature du système auquel ils appartenaient, où leur aspect figuratif n'avait jamais été chargé d'une fonction mimétique, même s'ils pouvaient en donner superficiellement l'illusion.

Si l'écriture hiéroglyphique égyptienne, comme tous les systèmes fondés sur l'idéogramme - car il en va de même de l'écriture cunéiforme mésopotamienne et du système chinois -, font intervenir l'image dans leur structure, c'est en effet d'une tout autre manière que ne l'ont imaginé les Grecs. Ce qui caractérise ces écritures est l'intégration étroite à leur système non seulement de formes graphiques plus ou moins réalistes selon les cas, mais des qualités tant matérielles que visuelles de leur support, indispensables à leur lecture. Intervient d'abord la matière de ce support - pierre, argile, papyrus, bois ou papier -, mais aussi la valeur sociale - publique ou privée, religieuse ou laïque - dont cette matière est elle-même investie. D'autre part les potentialités directionnelles multiples de l'espace offert à l'écriture peuvent être privilégiées sur la cohérence verbale du texte, soit que la répartition des signes ne tienne pas compte de la linéarité de l'oral, soit que, au contraire, elle permette d'introduire une logique visuelle dans la transcription du discours, comme en Égypte et en Chine, où des orientations opposées données aux mêmes éléments phrastiques contribuent à en modifier le sens. Enfin, et cette fonction gardera toute son importance jusque dans les alphabets sémitiques, c'est en substituant au principe de l'enchaînement temporel propre à la syntaxe verbale celui de la juxtaposition et de la simultanéité des termes qui caractérise l'espace plastique que sont choisis en dernier ressort les éléments linguistiques jugés pertinents à l'écrit. Ainsi s'explique que la propriété principale de l'idéogramme est qu'il ne possède pas de définition unique et figée - autrement dit, que ce n'est pas son éventuelle valeur de représentation qui fait de lui un signe d'écriture -, mais qu'il est un «signe flottant». Ce signe est, en effet, toujours susceptible de pouvoir assumer alternativement trois fonctions selon le contexte où il se trouve, celle de "logogramme", c'est-à-dire de signe renvoyant à un champ lexical donné - comme le mot «taon» désignant l'insecte qui porte son nom -, de «phonogramme» - comme les homophones «temps» et «tant» du mot "taon" -, ou de «déterminatif» (ou «clé» en chinois), comme l'indice purement visuel de la catégorie «insecte» 14 .

Née de l'image, l'écriture idéographique en a donc gardé les deux propriétés essentielles: elle ne repose pas sur l'absence d'un locuteur mais sur une présence, celle du lecteur, et sur l'ensemble d'observations et d'interrogations auxquelles celui-ci peut soumettre la surface où le texte se trouve inscrit, comme le fait le spectateur d'une image des différents éléments figures, espace, lignes et couleurs - qui la composent. L'écriture est fondée sur la lecture, une lecture qui n'est pas de l'ordre du déchiffrement de signes dont on attend qu'ils ressuscitent une parole devenue inaccessible, mais de la recherche raisonnée du sens, comme l'était celle des devins.

Seul l'alphabet grec, en transformant le texte écrit en un message codé, et codé phonétiquement, a rendu inutile au fonctionnement de l'écriture le support sur lequel elle se donne à lire. Mais cette dissociation de l'écriture et de la lecture, ce retour fictif à l'oral puisqu'il y manque la voix, a suscité l'invention d'une visibilité nouvelle du texte, confirmant qu'une telle visibilité demeurait indispensable à sa lecture comme elle l'avait été à celle de toutes les écritures précédentes: gloses marginales, orthographe, enluminure, variations stylistiques des lettres sont autant de témoignages de la réappropriation par l'alphabet de la surface d'où il se trouvait exilé. 
Ainsi, le raisonnement de Platon sur l'écriture, dont découle sa conception de l'icône, ne se justifie qu'eu égard à l'écriture telle qu'il la connaissait et la pratiquait lui-même, l'alphabet, une écriture où l'introduction de la notion de représentation, si elle pouvait convenir à la lettre conçue comme signe-son, trahissait en fait l'écriture et ses fondements iconiques. En s'appuyant sur une définition dynamique de l'icône, Peirce - et c'est un des intérêts majeurs de sa sémiotique - a fait échapper le hiéroglyphe à cette confusion et à ses pièges. Mais il a restreint ce dynamisme à l'élaboration d'un signe conçu comme une unité fixe et qui restait inspirée par l'alphabet. L'idéogramme, chez Peirce, ne "flotte» pas, il ne peut être défini par la mobilité de ses fonctions parce qu'il n'a pas attiré avec lui, dans le raisonnement du philosophe, cette surface dont l'apparence décidait seule, pourtant, de son sens ${ }^{15}$.

Un tel aveuglement pouvait-il être évité? Sans doute tient-il pour l'essentiel à l'orientation logique que Peirce a donnée à sa recherche et sur laquelle il n'est pas revenu, à la différence de Wittgenstein. L'expérience tentée par Mallarmé dans le Coup de dés ne nous permet cependant pas d'en douter: le recours à l'alphabet n'interdit pas de concevoir non seulement deux usages mais deux pensées différentes de l'écrit, selon qu'on l'envisage à partir de la ligne - ou du Vers, «ligne parfaite» - qui est celle de la voix, et par conséquent de l'alphabet, ou «selon une vision simultanée de la Page» qui relève du modèle idéographique. La description que Mallarmé nous donne de son poème pourrait être, en effet, à peu près celle d'un texte sumérien, égyptien ou chinois:

Le papier intervient chaque fois qu'une image, d'elle-même, cesse ou rentre, acceptant la succession d'autres et, comme il ne s'agit pas, ainsi que toujours, de traits sonores réguliers ou vers plutôt, de subdivisions prismatiques de l'Idée, l'instant de paraître et que dure leur concours, dans quelque mise en scène spirituelle exacte, c'est à des places variables, près ou loin $d u$ fil conducteur latent, en raison de la vraisemblance, que s'impose le texte.
Ce texte obéit à d'autres lois que celles auxquelles nous a habitués l'alphabet et son conditionnement logocentrique, mais il ne les rend pas caduques, se contentant seulement de faire apparaitre leur caractère relatif. Surtout, il permet enfin à l'écriture, en lui faisant assumer à nouveau sa pleine dimension spatiale, de retrouver la nécessité qui était celle de ses origines, offrir un domaine inédit à la pensée:

Le genre, que c'en devienne un comme la symphonie, peu à peu, à côté $d u$ chant personnel, laisse intact l'antique vers, auquel je garde un culte et attribue l'empire de la passion et des rêveries; tandis que ce serait le cas de traiter, de préférence [...] tels sujets d'imagination pure et complexe ou intellect: que ne reste aucune raison d'exclure de la Poésie. 16

L'audace de Mallarmé a consisté à combiner les préoccupations du linguiste avec celles du visionnaire, la dynamique du verbe aux intuitions erratiques de la lecture. Nul n'entre, en effet, dans le Coup de dés s'il n'est d'abord un lecteur. Ce n'était pas la seule blancheur de la page et ses constellations divinatrices qui avait convaincu le poète de la nécessité de déléguer à autrui l'autorité de son propre texte. Celles du mime et de la danse lui avaient révélé que la pensée naît d'autant plus pure de l'espace et de l'écriture des corps que la parole en est absente. Car telle est bien la "qualité», dont l'apparence a le privilège: faire qu'une surface ou un lieu ne soient pas le simple dépôt, arbitraire et provisoire, des signes, mais qu'ils en provoquent l'apparition, permettre d'inscrire dans la recherche verbale du sens une expérience visuelle qui lui est en principe étrangère.

Mais ni la logique ni l'alphabet ne tolèrent le mélange: Peirce n'a-t-il pas affirmé que «et, $\&$ et le son» n'étaient qu'un seul et même mot, niant ainsi toute pertinence «iconique» aux variations graphiques de l'écrit qu'il était pourtant le premier à constater? La pensée visuelle du signe, qui a sa source dans celle de l'apparence (ou plus généralement de l'écran 17), ne peut être qu'exclue a priori de toute sémiotique générale, quand bien même l'aurait-elle suscitée. 


\section{NOTES}

1. Ch. S. Peirce, Écrits sur le signe, rassemblés, traduits et commentés par G. Deledalle, Paris, Seuil, 1978, p. 35.

2. Platon, Timée, 28a-30a et Phèdre, 249d-250c.

3. Timée, $71 \mathrm{~d}-72 \mathrm{c}$ et Phèdre, 244b-245a.

4. Ibid., 45c.

5. Voir à ce sujet G. Simon, Le Regard, l'être et l'apparence dans l'optique de l'antiquité, Paris, Seuil, 1988.

6. Le Banquet, $215 \mathrm{~b}$.

7. La République VI, 510a, trad. R. Baccou, Paris, GF Flammarion, 1966, p. 267.

8. Ibid., 500c-501d, p. 257.

9. Ibid., 512a-517b, p. 273-275.

10. J.-F. Lyotard, Discours, Figure, Paris, Klincksieck, 1971, p. 271.

11. Phèdre, 275d-276a, trad. E. Chambry, Paris, Garnier Frères, 1964, p. 166.
12. Théétète, 202a.

13. Ibid., 203d-204b.

14. J'ai développé cette analyse dans L'Image écrite ou la déraison graphique, Paris, Flammarion, 1995. Voir également la section "Espaces de l'écrit» du Grand Atlas des Littératures, Paris, Encyclopaedia universalis, 1990.

15. Je me permets de renvoyer sur ce point à mon article sur «Écriture et iconicité", Cuadernos de filologia francesa $n^{\circ} 7$, Universidad de

Extremadura, Caceres, 1993, p. 37-43.

16. S. Mallarmé, "Observation relative au poème Un coup de Dés jamais n'abolira le Hasard», Cosmopolis, 1897.

17. J'ai tenté de démontrer dans le livre cité en note 14 que l'écriture était née de l'image dans la mesure où celle-ci était elle-même le produit d'une "pensée de l'écran " originelle. 